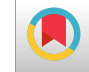

\title{
Functional Neurological Symptom Disorder in a Patient with Parkinson's Disease and Psychotic Features: A Case Report
}

\author{
Seyed Abolfazl Ghoreishi (iD) ${ }^{1, *}$ and Hoda As'adi ${ }^{2}$ \\ ${ }^{1}$ Department of Psychiatry and Social Factors Influencing Health Center and Metabolic Research Center, Zanjan University of Medical Sciences, Zanjan, Iran \\ ${ }^{2}$ Zanjan University of Medical Sciences, Zanjan, Iran \\ Corresponding author: Social Determinant of Health Research Center and Metabolic Disease Research Center, Zanajn University of Medical Sciences, Zanjan, Iran. Tel: \\ +98-9123199519, +98-2433544001, Fax: +98-2433534500, Email: sabgho@zums.ac.ir
}

Received 2020 March 02; Revised 2020 August 27; Accepted 2020 October 06.

\begin{abstract}
The conversion disorder (CD) or functional neurological symptom disorder is a diagnostic category used in some psychiatric classification systems, which often refers to the patients presenting with neurological symptoms. Parkinson's disease (PD) essentially affects non-motor and motor functions. The chronic use of levodopa, which is the primary treatment in this regard, has been reported to cause complications such as the wearing-off phenomenon. This problem may also increase the disease symptoms, as well as the patients' need to receive higher doses of the drug to remain symptom-free for a longer period. This case study aimed to describe a 47-year-old male patient with PD and psychotic features, the symptoms of which had progressed in a conversional setup relating to particular visitation times. According to the examinations, $C D$ was the main problem, which simulated the features of PD.
\end{abstract}

Keywords: Functional Neurologic Symptom Disorder, Conversion Disorder, Drug-Induced Psychosis, Parkinson's Disease

\section{Introduction}

The conversion disorder (CD) or functional neurological symptom disorder is a diagnostic category used in some psychiatric classification systems, which often refers to the patients presenting with neurological symptoms (e.g., numbness, blindness, paralysis or fits), which are not consistent with a well-established organic cause, may lead to significant distress, could be traced back to a psychological trigger, and cannot be explained by medical evaluation.

Parkinson's disease (PD) is essentially characterized by motor function symptoms in the form of resting tremor, rigidity, and bradykinesia. However, evidence suggests that the motor function symptoms are the 'tip of the iceberg' in terms of the clinical manifestations of PD. In addition to motor function symptoms, PD is characterized by several non-motor symptoms, including cognitive decline, psychiatric disturbances (e.g., depression, psychosis, and impulse control), sleep difficulties, autonomic failures (e.g., gastrointestinal, cardiovascular, urinary, and thermoregulation complications), and pain syndrome (1).

In the early stages of PD, numerous patients are unaware of their movement reduction. The first signs of movement and expression deficits are often detected by the patient's spouse as it is observed that activities such arm swing while walking, dressing, and eating are notably slowed. Once the manual skills and fine works are decelerated, the patient becomes aware of the disability (2). The communication problems in patients with PD lead to less assertiveness, thereby rendering them socially withdrawn and passive (2). In the course of PD treatment, medications could induce psychotic symptoms, such as hallucination and delusion, as well as mood disorders, cognitive impairment, and sleep disturbances (3).

$\mathrm{CD}$ refers to the conditions associated with neurological disabilities without a definite neurological cause. From a different perspective, it is a type of hysteria that could be manifested by the temporary loss or change in the motor or sensory functions. CD motor symptoms mimic syndromes such as paralysis, ataxia, dysphagia, and the seizure disorder (pseudo-seizures), while the sensory symptoms resemble those of neurological deficits such as blindness, deafness or anesthesia (4). In patients with $C D$, the mean volume of the right and left basal ganglia and the right thalamus have been reported to be smaller compared to the controls with normal volumes (5).

\section{Case Presentation}

The patient was a 47-year-old male who was a known case of young onset PD for approximately 14 years. The signs and symptoms included tremor, muscle spasticity, 
rigidity, dyskinesia, and festinating gait. Within the first 3 - 4 years of diagnosis, the patient avoided medication due to weak coping and poor compliance. However, he accepted and began medication gradually with his improved disability. The patient received treatment with levodopa$\mathrm{C}$ forte and pramipexole. During the treatment, he recovered dramatically from tremor, dyskinesia, and rigidity and was able to resume his job and partake in activities such as driving.

The patient remained under the casual observation of a neurologist. Over the years and with the progression of PD, he changed his medication instructions for better daily function. The patient reduced the levodopa intervals knowing that by passing time before the next dose, the serum dopamine would decrease and cause symptoms flare-up. The prescribed medication for the patient was levodopa-C forte q6h and pramipexole $0.7 \mathrm{q} 6 \mathrm{~h}$, but he personally made a schedule of using $1 / 2$ levodopa-C forte every two hours, every one hour, and sometimes every half an hour. In addition, pramipexole was taken as 1/2 tab every 3 - 4 hours.

Due to his irregular medication use, the patient developed a psychotic disorder. He had paranoid delusions about his family, grandiosity, and hyper religious delusions (e.g., close relation with God), and reference delusions (e.g., hearing the mention of his name on TV). The other symptoms of the patient were expansive mood, talkativeness, free-spending, increased libido, and distractibility.

The patient was admitted, and antipsychotic treatment with clozapine was initiated. After two weeks, the signs and symptoms gradually disappeared. The treatment continued with clozapine TDS ( $25 \mathrm{mg}$ ), and the antiPD medications were administered at longer intervals, including $1 / 2$ tabs of levodopa-C forte every 1 - 2 hours to $1 / 2$ tabs every four hours. As a result, the dyskinesia, tremor, and movement instability of the patient improved significantly. After 10 days, we changed levodopa-C forte usage to one tab every eight hours, which caused no significant movement impairment.

During admission and the daily visitations, some psychogenic signs were detected in the patient's manner, such as ataxia and astasia-abasia, which indicated the incidence of $\mathrm{CD}$. The patient was transferred to the visitation room by wheelchair, stating that he had difficulty walking straight. After a while, the patient was distracted by the clinician's questions and began to stand up and walk normally, claiming that he was experiencing the effects of the medication.

Evidently, the disability condition of the patient stemmed from a type of hysteria although he did not present with hysteric symptoms in the random observations of the residents. Moreover, ataxia was not reported by the residents, and the nursing staff did not detect any unusual behaviors (except psychosis) in their hourly checking or medication time. It seems that the conversional actions of the patient were mostly observed in the visitations of the main clinician, and the patient remained nearly symptom-free at other times. Notably, primary gains for the patient were denied in the interviews with her family, as well as the daily visits. Furthermore, the CD diagnosis of the patient was confirmed by a psychiatrist.

The behaviors of the patient in medication use at home had an obsessive feature. Accordingly, he searched multiple sites to find disease indications, pharmacokinetics, directions of use, and side-effects of the anti-PD drugs, which finally led to paranoia and control delusions.

\section{Discussion}

PD with psychosis tends to appear later in the course of the disease, and disease duration represents an important risk factor for PD development. Among the other risk factors are the use of anti-PD medications, sleep disturbances, old age, dementia and/or depression, disease severity, and cognitive impairment. Recent studies have aimed to explain the complex pathophysiology of PD psychosis, which is now known to involve an overlap between extrinsic, medication-related, and intrinsic disease-related components (6).

A case series performed by Factor S. A. et al. regarding the cognitive correlates of hallucinations and delusions in PD indicated that the frequency or severity of the hallucinations were significantly correlated with the lower scores of attention, language, memory, visuospatial ability, and executive functioning, while delusions may not share the same associations with dementia as hallucinations (7).

The symptoms of the CD could be detected due to a psychological conflict as these symptoms are abruptly observed after a stressful experience. In addition, individuals are considered high-risk for the CD if they have a medical illness or other psychological problems associated with various personality disorders or dissociative disorders. In the present study, we did not obtain a case report in which PD symptoms signify the CD. Such problems in these patients could be attributed to hysteria and unintentional behaviors. In other words, frequent and regular episodes of symptom flare-up and disability tend to occur under particular circumstances. Therefore, it is advised that in the unusual cases of PD, clinicians consider similar conditions in which the CD might mimic the original disease. If specialists initiate the treatment, the underlying disease is likely to exacerbate and increase anti-PD medication, thereby leading to the deterioration of the associated complications.

Since our attention was drawn to the untrue and misleading symptoms of the patient, we started to consider them as inside conflicts and unconsciousness. Therefore, 
we avoided increasing the medication doses and even prescribed lower doses. The early PD symptoms were more important in the patient than the symptoms of psychosis. In addition, the $\mathrm{CD}$ of the patient was observed to relief the secondary gain during the daily visitations of the specialist, along with the exacerbation of the clinical and behavioral symptoms in order to index the PD symptoms. The specialist shifted the medication regimen of the patient to anti-PD drugs, which might have caused the psychotic symptoms. In order to interrupt this defective cycle to reduce the symptoms of the patient, it is essential to reduce the dose of anti-PD drugs and disregard the patient's request to increase the dose of the drugs as in the current case, no attention was paid to the clinical symptoms that exacerbated by the patient professionally and the reduction the primary and secondary gains of the patient. At the next stage, antipsychotic medication (clozapine) was observed to have the least aggravating effects on the underlying disease at the least effective dose in the treatment regimen. Fortunately, the changes of previous medication and approaches improved both the PD and psychosis symptoms. Finally, the patient was discharged in good general condition, and the disease was controlled.

\subsection{Conclusions}

Based on this case report, the following suggestions are made:

1) Use of minimal anti-PD drugs with standard doses;

2) Structural and psychological interventions to reduce the primary and secondary gains of PD patients;

3) Improving the self-confidence of patients;

4) Clozapine treatment to control the symptoms of psychosis.

\section{Footnotes}

Authors' Contribution: Contribution of the authors as mentioned below with their responsibility in the research.
Seyed Abolfazl Ghoreishi did conception and design of study, critical revision, and final approve of study. Hoda As'adi did collection and possession of data and study concept and design.

Conflict of Interests: There is no conflict of interest.

Funding/Support: We had not funding support.

Informed Consent: All the stages of examination, history taking, and treatment were performed with the complete awareness and satisfaction of the patient, and informed consent was also provided by the patient.

\section{References}

1. Grover S, Somaiya M, Kumar S, Avasthi A. Psychiatric aspects of Parkinson's disease. J Neurosci Rural Pract. 2015;6(1):65-76. doi: 10.4103/09763147.143197. [PubMed: 25552854]. [PubMed Central: PMC4244792].

2. Poewe W, Seppi K, Tanner CM, Halliday GM, Brundin P, Volkmann J, et al. Parkinson disease. Nat Rev Dis Primers. 2017;3:17013. doi: 10.1038/nrdp.2017.13. [PubMed: 28332488].

3. Ffytche DH, Creese B, Politis M, Chaudhuri KR, Weintraub D, Ballard C, et al. The psychosis spectrum in Parkinson disease. Nat Rev Neurol. 2017;13(2):81-95. doi: 10.1038/nrneurol.2016.200. [PubMed: 28106066]. [PubMed Central: PMC5656278].

4. Ali S, Jabeen S, Pate RJ, Shahid M, Chinala S, Nathani M, et al. Conversion Disorder- Mind versus Body: A Review. Innov Clin Neurosci. 2015;12(5-6):27-33. [PubMed: 26155375]. [PubMed Central: PMC4479361].

5. Roelofs JJ, Teodoro T, Edwards MJ. Neuroimaging in Functional Movement Disorders. Curr Neurol Neurosci Rep. 2019;19(3):12. doi: 10.1007/s11910-019-0926-y. [PubMed: 30747347]. [PubMed Central: PMC6373326].

6. Chang A, Fox SH. Psychosis in Parkinson's Disease: Epidemiology, Pathophysiology, and Management. Drugs. 2016;76(11):1093-118. doi: 10.1007/s40265-016-0600-5. [PubMed: 27312429].

7. Factor SA, Feustel PJ, Friedman JH, Comella CL, Goetz CG, Kurlan R, et al. Longitudinal outcome of Parkinson's disease patients with psychosis. Neurology. 2003;60(11):1756-61. doi: 10.1212/01.wnl.0000068010.82167.cf. [PubMed: 12796526]. 